AUTOIMMUNE DIABETES

\section{NKG2D receptor: a therapeutic target?}

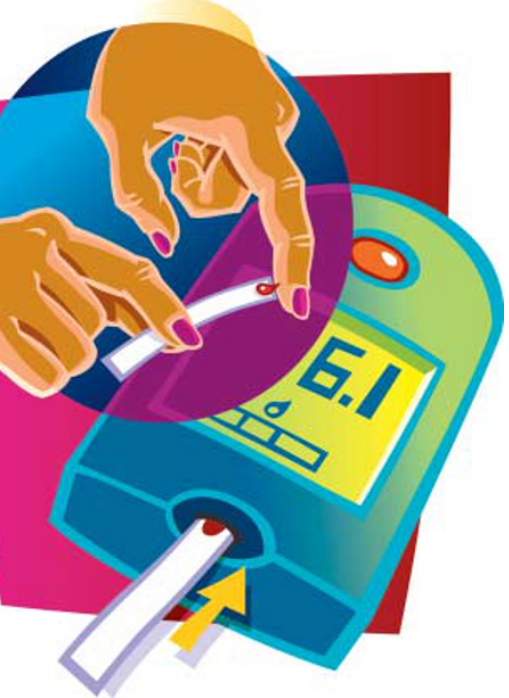

Type 1 diabetes is an autoimmune disease typically characterized by a long preclinical stage in which insulinproducing $\beta$ cells in the pancreatic islets are destroyed by autoimmune $\mathrm{T}$ cells. In the June issue of Immunity, Lewis Lanier and colleagues use the non-obese diabetic (NOD) mouse to show that the ligand retinoic acid early inducible (RAE)-1, which is present in pre-diabetic islets, activates the receptor NKG2D, which is expressed on the surface of a subset of T cells and natural killer (NK) cells; furthermore, this interaction is essential for disease progression. When treated during the pre-diabetic stage, a nondepleting anti-NKG2D antibody completely prevented disease.

The NOD mouse is widely used as a model of human type 1 diabetes. Usually, inflammation of the islets is observed 10-20 weeks before T-cell destruction of the insulin-producing cells; this process and the time delay is not well understood. NKG2D is a costimulatory receptor expressed on activated NK cells and certain groups of T cells, and there is evidence that it might carry out surveillance against cancer and infections. The ligands of NKG2D, including RAE-1, are known to be molecules whose expression is triggered by oncological transformation and infection with viral and bacterial pathogens. Costimulatory molecules on $\mathrm{T}$ cells and other immune cells are crucial for the autoimmune response. Expression of RAE- 1 family members is strictly regulated in normal cells, and little is expressed on healthy adult tissues. However, the authors found that RAE-1 was expressed on islet cells in NOD mice, but not in other strains such as $\mathrm{C} 57 \mathrm{BL} / 6$ or $\mathrm{BALB} / \mathrm{c}$. Expression was also detected in the pancreases of adult immune-deficient NOD mice (which lack T cells and do not develop diabetes), indicating that RAE-1 expression is independent of ongoing autoimmune response.

To demonstrate the role of NKG2D in the progression of diabetes, the authors transferred T cells from diabetic NOD mice into immune-deficient NOD mice. Diabetes developed in all control mice, but not those treated with antiNKG2D antibodies. The authors showed that the therapy impaired the clonal expansion and function of autoreactive T cells in the pancreas, although it did not prevent the initial activation of the T cells.
ANTICANCER DRUGS

\section{Taking out the chaperone}

Targeting the molecular chaperone Hsp90 is emerging as a promising anticancer strategy, as highlighted in two recent papers in Chemistry and Biology on a class of Hsp90 inhibitors. In contrast to other 'targeted' anticancer agents, which are typically aimed at a single cancer-associated molecular alteration, these Hsp90 inhibitors have multiple antitumour-specific effects, meaning that they might be active in a considerably broader range of cancers.

Molecularly targeted therapies have now been the focus of anticancer drug development programmes for several years, and have proved highly successful in a few cases in which the cancer is dependent on the targeted oncoprotein, as, for example, is the case with imatinib (Gleevec; Novartis). However, most tumours are characterized by multiple molecular alterations, which are specific to the tumour. So, targeting just one alteration might be insufficient, and is only likely to show significant success for the subset of tumours in which the targeted protein has a key role.

How might these problems be circumvented? One strategy that has been proposed is to target the machinery that allows cancer cells to function despite the abnormalities that they carry. Hsp90 is a key component of this machinery, as it is required for the stability and functioning of a number of mutated, chimeric and overexpressed signalling proteins that promote the growth and/or survival of cancer cells. Furthermore, it seems that differences between the activation state of Hsp90 in tumours and normal cells allow Hsp90 inhibitors to show good selectivity for tumour cells.

However, Hsp90 inhibitors reported so far - which are primarily natural products or natural product derivatives - have not yet lived up to the promise of their target, largely owing to problems with bioavailability, solubility and toxicity. The structural complexity of the natural products makes the search for related compounds that are more drug-like very challenging, and so researchers have been looking for new families of Hsp90 inhibitors without these limitations.

In the first of the two papers, Chiosis and colleagues present $\mathrm{PU} 24 \mathrm{FCl}$, which is a representative of the first class of designed
Hsp90 inhibitors. This compound inhibited the growth of a wide range of cancer cell lines in vitro, and these effects correlated with its effects on Hsp90 client proteins thought to be involved in cancer cell growth and survival. And in mice bearing MCF-7 breast-cancer xenografts, $\mathrm{PU} 24 \mathrm{FCl}$ selectively accumulated in tumour tissue, and again showed a dosedependent effect on Hsp90 client proteins. Finally, in a 30-day study of antitumour efficacy in the MCF-7 xenograft model, treatment with $\mathrm{PU} 24 \mathrm{FCl}$ resulted in a $72 \%$ reduction in tumour burden compared with a control group.

The second paper, by Wright et al., reports crystal structures of the $\mathrm{N}$-terminal domain of $\mathrm{Hsp} 90$ complexed with several inhibitors in the same class as PU24FCl. These structures allowed the authors to develop a detailed explanation for the observed affinity of members of this class for Hsp90, and should prove valuable in the design of further compounds to fully realize the potential of Hsp90 inhibition as an anticancer strategy.

\section{Peter Kirkpatrick}

(1) References and links ORIGINAL RESEARCH PAPERS Vilenchik, M. et al. Targeting wide-range oncogenic transformation via PU24FCl, a specific inhibitor of tumor Hsp90. Chem. Biol. 11, 787-797 (2004) | Wright, L. et al. Structure-activity relationships in purine-based inhibitor binding to HSP9O isoforms. Chem. Biol. 11, 775-785 (2004) FURTHER READING Capdeville, R. et al. Glivec (STI571, imatinib), a rationally developed targeted anticancer drug. Nature Rev. Drug Discov. 1, 493-502 (2002) 
These findings are important because unlike other treatments reported in the NOD mouse this therapy is effective when administered relatively late in disease progression. Interestingly, NKG2D has been detected on T cells — isolated from the synovial tissue of rheumatoid arthritis patients - in the presence of one of its ligands, which raises the posibility that this receptor could play a detrimental role in other autoimmune diseases.

Melanie Brazil

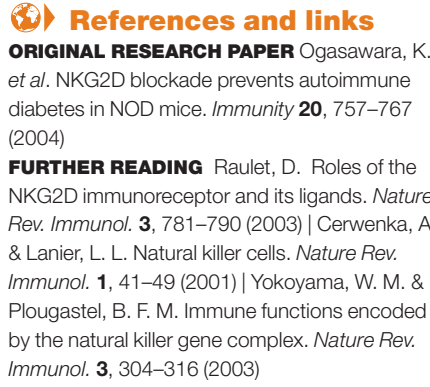

Immunol. 3, 304-316 (2003)

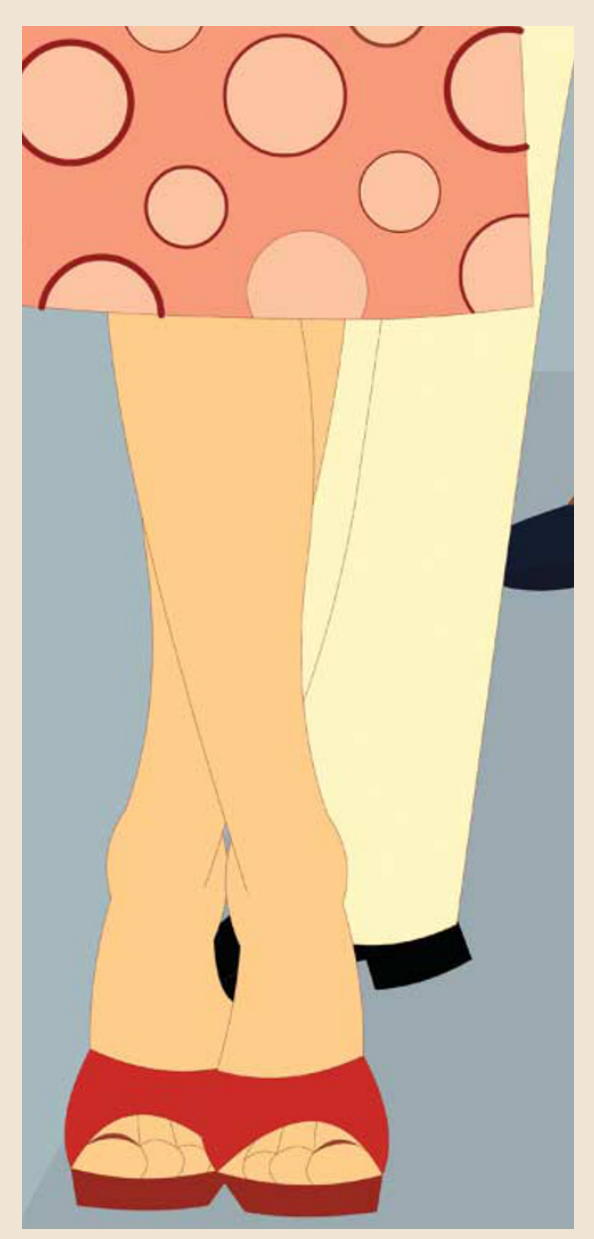

\section{Growing concern for tamoxifen}

Breast tumours that recur after treatment with the anti-oestrogen tamoxifen might result from a change in the effect of the drug that turns it from an antagonist of the oestrogen receptor into an agonist that promotes cancer cell growth. Writing in Cancer Cell, Rob Michalides and colleagues report that phosphorylation of oestrogen receptor- $\alpha$ (ER- $\alpha$ ) by protein kinase A (PKA) allows tamoxifen to bind but obliterates its antagonistic effect. Their findings mean that it will be possible to screen patients for activated PKA, which will indicate whether they would be more successfully treated with tamoxifen or a pure anti-oestrogen such as ICI-182780 (Fulvestrant/Faslodex).

Tamoxifen is a very effective treatment for oestrogen-receptor-positive $(\mathrm{ER}+)$ breast cancer that has few side effects and even beneficial properties such as promoting bone growth. However, $50 \%$ of recurrent ER+ breast cancers are resistant to tamoxifen. One of the proposed mechanisms for this resistance is phosphorylation of ER- $\alpha$ by PKA. Anti-oestrogens such as tamoxifen and ICI- 182780 act by binding to ER- $\alpha$ and causing a conformational change that prevents the recruitment of co-activators to ER- $\alpha$.

A recombinant ER- $\alpha$ was constructed that contained a different variant of green fluorescent protein at the amino and carboxyl termini to measure changes in the fluorescence resonance energy transfer (FRET) signal that occurs when the relative position of each fluorophore is altered by a change in receptor conformation. Using this assay, the authors studied the effect on receptor conformation of several factors that induce resistance to tamoxifen (SRC1, cAMP and cyclin D1).

FRET analysis showed that CAMP prevented tamoxifen from causing a conformational change of ER- $\alpha$, whereas SRC1 and cyclin D1 had no effect with or without cAMP. Not only did cAMP prevent tamoxifen antagonist action, it also caused the antioestrogen to behave as an agonist and upregulated ER- $\alpha$-responsive reporter genes. By contrast, cAMP could not overcome ICI-182780 antagonism of ER- $\alpha$. This is related to the fact that tamoxifen is a selective oestrogen receptor modulator (SERM) that behaves as either an agonist or antagonist depending on the cellular context, whereas ICI-182780 is a full antagonist.

The authors then investigated the mechanism of cAMP-induced resistance by studying the FRET pattern after exposure to forskolin, which stabilizes the generation of CAMP and therefore the activation of PKA. Forskolin prevented tamoxifen-induced

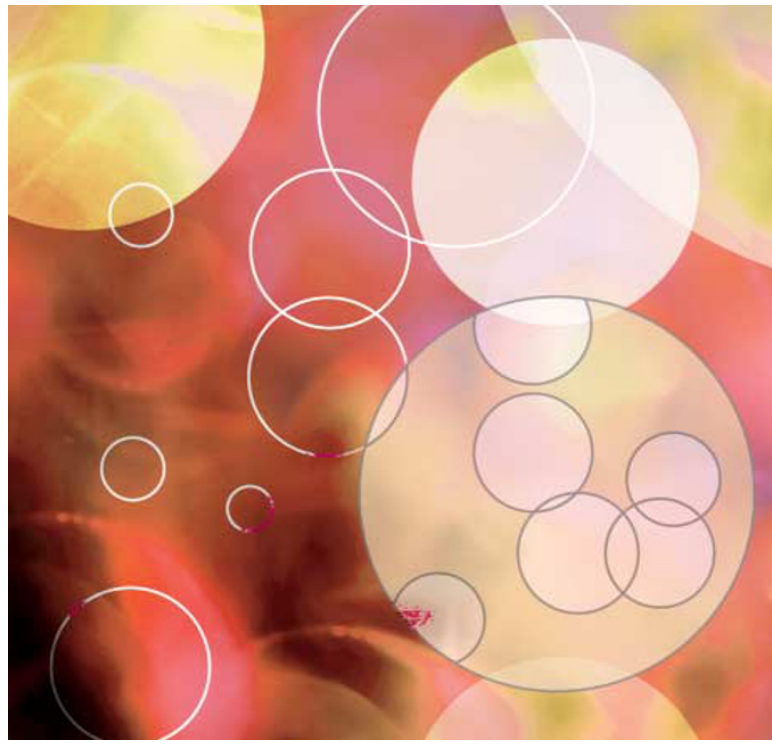

conformational change but had no effect on ICI182780. Even when a tenfold molar excess of ICI-182780 was added after tamoxifen treatment, no change in conformation was observed, which demonstrates that tamoxifen still binds to ER- $\alpha$ but is unable to induce the inhibitory conformation.

Mutational analysis of consensus PKA phosphorylation sites in ER- $\alpha$ showed that Ser305, which is located in the hinge region, is necessary for forskolin to prevent tamoxifen-induced conformational change, and indicates that PKA renders ER- $\alpha$ resistant to antagonism by tamoxifen.

To further dissect the role of the PKA pathway in tamoxifen resistance, the authors studied 70 $\mathrm{ER}+$ breast cancers isolated before tamoxifen treatment, 20 of which were later classed as tamoxifen-resistant. Gene expression analysis showed that tamoxifen-resistant breast tumours have downregulated PKA-RI- $\alpha$ (the PKA negative regulatory subunit). Correspondingly, RNA interference knockdown of PKA-RI- $\alpha$ induced ER- $\alpha$ resistance to tamoxifen, but not ICI-182780. Furthermore, T47D cells, which depend on oestrogen for growth, continued to grow in the presence of tamoxifen when PKA-RI- $\alpha$ was downregulated, whereas ICI-182780 retained its inhibitory effect on growth. This agonist action of tamoxifen induced by PKA explains the regression of tumours observed in tamoxifen-resistant patients when tamoxifen treatment is withdrawn. Selection of patients for anti-oestrogen treatment according to PKA activity should improve the success rate of tamoxifen treatment and prevent the further outgrowth of resistant tumour cells.

Joanna Owens

6) References and links ORIGINAL RESEARCH PAPER Michalides, R. et al. Tamoxifen resistance by a conformational arrest of the estrogen receptor $\alpha$ after PKA activation in breast cancer. Cancer Cell 5, 597-605 (2004) WEB SITE

Rob Michalides Lab: http://www.nki.nl/nkidep/h4/michalides/ michalides.htm 